\title{
ANALYSIS OF $4 f$ LEVEL IN SAMARIUM-RICH MBE GROWN CdSmTe SAMPLE
}

\author{
K. Szamota-Sadowska ${ }^{a}$, Z. Golacki ${ }^{a}$, B.A. Oreowski ${ }^{a}$, R. Boyn $^{b}$ \\ AND R.J. JOHNSON ${ }^{c}$ \\ ${ }^{a}$ Institute of Physics, Polish Academy of Sciences \\ Al. Lotników 32/46, 02-668 Warsaw, Poland \\ ${ }^{b}$ Humboldt-Universität zu Berlin, Institut für Physik \\ Invalidenstr. 110, 10115 Berlin, Germany \\ 'II Institut für Experimentalphysik, Universität Hamburg, 22761 Hamburg, Germany
}

Electronic states of $4 f$ samarium ions were investigated by photoemission spectroscopy in samarium-rich CdSmTe sample obtained by MBE. The photon energy of synchrotron radiation allowed to investigate Fano-type resonance and antiresonance. The energy distribution curve spectra were attributed to the Sm $4 d-4 f$ transition. The shape of the constant initial states spectra was compared with this one obtained for atomic samarium.

PACS numbers: 73.20.At, 79.60.-i, 79.60.Bm

\section{Introduction}

The influence of rare-earth ions on electronic structure of II-VI wide gap semiconductors is recently under investigation because of its interesting optical properties. On the other hand, application of molecular beam epitaxy (MBE) technique allows to obtain a new quality class of mixed crystals: II-VI compounds doped with rare earth. It should be mentioned that other standard techniques for obtaining the bulk crystals are not suitable for this purpose because of differences in the crystallographic structure between zinc blende $\mathrm{CdTe}$, and rock salt for rare-earth telluride.

In many compounds the problem of small solubility of rare-earth atoms in II-VI compounds limits the number of the possible type of measurements. Samarium is an exceptional rare-earth element, which gives possibility to obtain by MBE technique a good quality crystal $\mathrm{Cd}_{1-x} \mathrm{Sm}_{x} \mathrm{Te}$ with high concentration of samarium.

The photoemission spectroscopy as a surface sensitive technique for examination of surface states is very useful for investigation of such type of the crystals. In the present paper the first examination of $\mathrm{CdSmTe}$ thin layer by means of resonant photoemission experiment is presented. The RE $4 f$ orbitals spectra are difficult for interpretation because of high localisation and strong correlation of the electrons. 


\section{Experiment}

The experiment of resonant photoemission was performed on thin layer of $\mathrm{CdSmTe}$, grown by MBE on CdTe/ZnTe buffer layer crystallised on GaAs (100) substrate. It was prepared in the Institute of Physics, Humboldt-Universität zu Berlin. The sample was rich in samarium with concentration around $10^{22} \mathrm{~cm}^{-3}$. During the growth process, when the concentration of samarium was above $10^{21} \mathrm{~cm}^{-3}$, the special effect of transition from zinc blende structure into rock salt structure was observed. The detailed sample preparation procedure is described in Ref. [1].

The reported experiment was carried out in a photoemission spectrometer attached to the beam line Flipper II of the Storage Ring DORIS II in HASYLAB at DESY in Hamburg, Germany. The spectra were measured in two photoemission techniques: constant initial states (CIS) and energy distribution curve (EDC). The first one gives information about contribution of Sm electrons to the density of states of particular region of the valence band. If the obtained curve has Fano-type profile one can find value of transition characteristic of Fano resonance and antiresonance. The EDC mode allows to investigate the occupied state in the valence band.

\section{Results}

The EDC spectra of CdSmTe thin layer are presented in Fig. 1. The spectra are chosen in order to show significant changes of the density of states as a function of photon energy. The features of the obtained curve correspond to the $\mathrm{SmTe}$ structure - it is difficult to distinguish the density of states of CdTe, probably, because of smaller than for Sm intensity of its transition in this energy region. Each of the photon energy value is marked on the spectrum. The zero binding energy position was localised at the Fermi level. The $a, b$ structures (in Fig. 1) were assigned to the dominant influence of $4 f$ shell while the influence from $5 p$ shell were marked by $c$ and $d$. The doublet character of the observed structures is originated from spin-orbit splitting.

On the bottom spectrum, the structures are marked for the following binding energies: small shoulder near $-2.014 \mathrm{eV}$ and the peaks $a=-5.6 \mathrm{eV}, b=-8.56 \mathrm{eV}$, $c=-20.5 \mathrm{eV}, d=-25.9 \mathrm{eV}$. The intensity of the peak marked by $b$ changes for different values of photon energies. One can see that for the photon energy equal to $135.4 \mathrm{eV}$ only small shoulder is visible but for the photon energy equal to $140 \mathrm{eV}$, the maximum intensity of $b$ peak was observed. For higher photon energy, equal to $143 \mathrm{eV}$, the small decrease in intensity was observed also for peak marked by $d$. The highest intensity was observed for energy equal to $135.4 \mathrm{eV}$, but when photon energy was equal to $14 n \mathrm{eV}$, a decrease in intensity of the structure was observed. And at least, at photon energy equal to $143 \mathrm{eV}$ the $d$ peak has more smoothing character.

The splitting of $4 f^{-1}$ final states equal to $2.9 \mathrm{eV}$ and the splitting of $5 p^{-1} \mathrm{fi}-$ nal states equal to $5.4 \mathrm{eV}$ are comparable with splitting of final states of Sm atomic sample [2] (2.7 $\mathrm{eV}$ and $6.1 \mathrm{eV}$ respectively). Taking into account these properties and localised character of $f$ shell, one can assign the structures mentioned above to the following transition basing on the results for atomic samarium [2]. The 


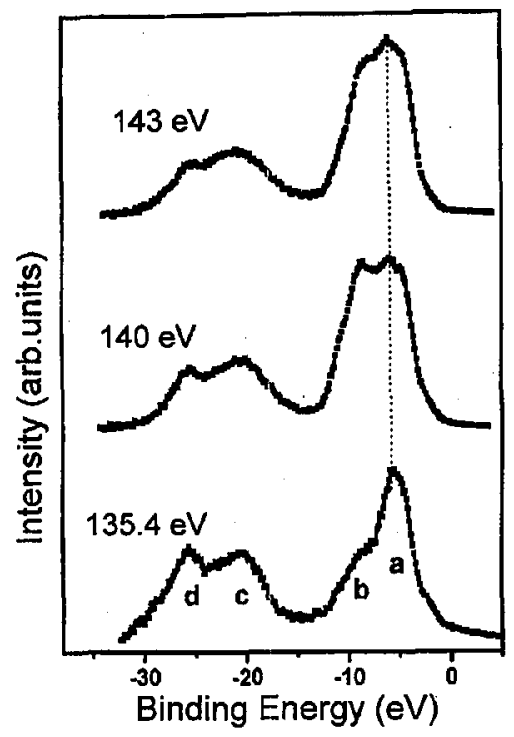

Fig. 1. Energy distribution curves of CdSmTe thin layer collected for photon energy: $135.4 \mathrm{eV}, 140 \mathrm{eV}, 143 \mathrm{eV}$ respectively. The contribution of $4 f$ shell were marked by $a, b$ while of $5 p$ by $c, d$. The zero energy position was located at the Fermi level.

structure marked as $a$ is assigned to the $\operatorname{Sm} 4 f^{6} 6 s^{2}{ }^{7} F \rightarrow 4 f^{5} 6 s^{2}{ }^{6} H{ }^{6} F$, while marked by $b$ is caused by the $\operatorname{Sm} 4 f^{6} 6 s^{2}{ }^{7} F \rightarrow 4 f^{5} 6 s^{2}{ }^{6} P$ transition. The structure marked by $c$ is attributed to the final state $5 p_{3 / 2}^{5} 4 f^{6} 6 s^{2}$ while marked by $d$ to the $5 p_{1 / 2}^{5} 4 f^{6} 6 s^{2}$. It should be mentioned that the binding energy of the above peaks is shifted to the smaller binding energy in comparison with data for atomic samarium [2] but in comparison with the result for $\mathrm{SmTe}$ these values of binding energies are shifted to higher energies [3].

Figure 2 shows the spectra obtained by CIS technique. The spectrum marked by $\mathrm{A}$ was measured for initial energy equal to $5.5 \mathrm{eV}$, while this one marked by $\mathrm{B}$ was measured for initial energy equal to $25.5 \mathrm{eV}$. The first energy corresponds to the density of states of $4 f$ electrons while the second one to the density of states of $5 p$ electrons.

The many-body character of transition in samarium compound was visible in these two spectra as a superposition of typical Fano-line shapes. The line presents effect of Fano-type fitting procedure performed for main peak. The partial cross-section are plotted at bottom part of each spectrum. In the both spectra one can observe structures with the following maxima:

Spectrum A

\begin{tabular}{l|l|r|r|r|r}
\hline $129 \pm 1$ & $134 \pm 1$ & $\begin{array}{r}137.9 \pm 0.2 \\
\text { Spectrum B }\end{array}$ & $\begin{array}{c}140.4 \pm 0.2 \\
\text { S }\end{array}$ & $143.4 \pm 0.2$ & $151.2 \pm 0.2$ \\
\end{tabular}

\begin{tabular}{l|l|l|l|l|l}
\hline $131 \pm 0.4$ & $134 \pm 0.1$ & $138.8 \pm 0.2$ & $140.7 \pm 0.2$ & $143.3 \pm 0.2$ & $151.1 \pm 0.2$
\end{tabular} The values obtained from fitting procedure are printed in italic font. 


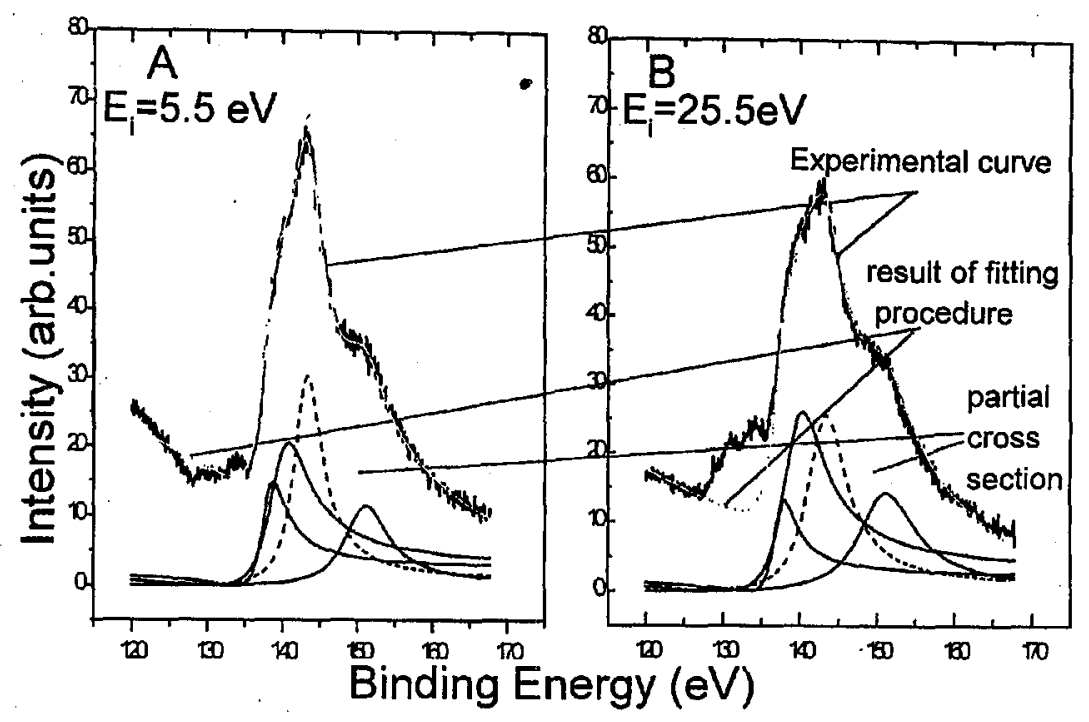

Fig. 2. Spectra collected in constant initial state mode. For two values of initial energy: $E_{\mathrm{i}}=5.5 \mathrm{eV}$ spectrum marked by $\mathrm{A}$ and for $E_{\mathrm{i}}=25.5 \mathrm{eV}$ marked by $\mathrm{B}$.

Both spectra have shape similar to the one obtained for $\mathrm{Sm}$, however one can observe differences in region around $138 \mathrm{eV}$. In both spectra an increase in the shoulder in this energy region is observed, however this structure is more visible in spectrum B. As it is visible also from comparison of fitting value, this structure does not influence the position of main peak. The other small difference between these two spectra is observed in intensity of two peaks lying on the left side of the main maximum.

An interesting conclusion appears when we combine EDC and CIS spectra together. When we look at EDC spectrum collected for $140 \mathrm{eV}$ and take into account a small structure appearing at the same photon energy in CIS spectrum we can connect appearance of these two structures from the same shell. The increase in peak marked by $b$ between values of photon energy from $135.5 \mathrm{eV}$ to $140 \mathrm{eV}$ in the EDC spectrum and the appearing of the shoulder near to $138 \mathrm{eV}$ in CIS spectrum lead to the conclusion that this structure originates from the influence of $4 f$ electrons. One can suppose that this structure corresponds to the $4 d-4 f$ transition mixed with influence of $5 p^{-1}$ photoionization cross-section which is seen as increase in $d$ peak on EDC spectrum for photon energy equal to $140 \mathrm{eV}$.

\section{Summary}

The obtained spectra were compared with the spectra measured for atomic samarium. The Sm $4 f$ electrons found to be located at the top part of the CdTe valence band $(a=-5.6 \mathrm{eV}$ and $b=-8.56 \mathrm{eV}$ - Fig. 1). The Sm $5 p$ electrons binding energies were found as two spin-orbit split peaks with binding energies: $-20.5 \mathrm{eV},-25.9 \mathrm{eV}$ relatively for $c$ and $d$ peak (Fig. 1). 
CIS spectra (Fig. 2) show the maximum value obtained for $143.3 \mathrm{eV}$. The CIS spectra possess rich structure character well corresponding to the rich structure of absorption spectra of $\mathrm{Sm} 4 f$ electrons [2]. The interpretation of the obtained results needs application of highly advanced theory as the $\mathrm{Sm} 4 f$ orbital possesses localised character with the strongly correlated electrons.

\section{Acknowledgments}

This work was supported by the Committee for Scientific Research (Poland) by grant No. 2 P03B 08910.

\section{References}

[1] D. Wruck, R. Boyn, L. Parthier, F. Henneberger, Semicond. Sci. Technol. 12, 179 (1997).

[2] M. Richter, M. Meyer, M. Pahler, T.H. Prescher, E.v. Raven, B. Sonntag, H.E. Wetzel, J. Phys. B 11, 5666 (1986). 